\title{
Endoftalmite: uma análise de 58 casos
}

\author{
Endophthalmitis: an analysis of 58 cases
}

Tarciso Schirmbeck (1)

Erasmo Romão ${ }^{(2)}$

Maria de Lourdes Veronese Rodrigues ${ }^{(3)}$

José Fernando de Castro Figueiredo ${ }^{(4)}$

\section{RESUMO}

Objetivo: Foram estudados 58 casos com diagnóstico de endoftalmite no intuito de se obter as características regionais desta patologia. Avaliaram-se os fatores predisponentes, quadro clínico, exames complementares, tratamento e resultado funcional.

Métodos: Foi feita a análise retrospectiva dos prontuários dos pacientes atendidos no HCFMRP-USP, com diagnóstico de endoftalmite, no período de 1993 a 1998.

Resultados: Trauma e cirurgia foram os principais fatores desencadeantes $(39,05 \%$ cada). $O$ quadro clínico predominante foi dor e diminuição da acuidade visual associado a hiperemia conjuntival e hipópio. Em 70,48\% dos casos colheu-se cultura, sendo o resultado positivo em $85,36 \%$ das amostras. $O$ agente etiológico mais freqüente foi o $S$. aureus $(26,08 \%)$, seguido do $S$. epidermidis e $P$. aeruginosa $(13,04 \%$ cada $)$. Todos agentes isolados foram sensíveis à vancomicina e à ceftazidima. $\mathrm{Em} \mathbf{6 5 , 5 1 \%}$ dos casos a acuidade visual final foi ausência de percepção luminosa.

Conclusões: Os resultados sugerem algumas alterações a serem efetuadas para se melhorar o prognóstico visual destes casos, como o uso de vancomicina e ceftazidima intra-ocular.

Palavras-chave: Endoftalmite; (quadro clínico, microbiologia, tratamento).
Departamento de Otorrinolaringologia e Oftalmologia

(1) Aluno de graduação da Faculdade de Medicina de Ribeirão Preto - Universidade de São Paulo (FMRP-USP).

Professor Associado ao Departamento de Otorrinolaringologia e Oftalmologia da FMRP-USP.

(3) Professora Doutora do Departamento de Otorrinolaringologia e Oftalmologia da FMRP-USP.

Professor Doutor do Departamento de Clínica Médica da FMRP-USP.

Endereço para correspondência: Erasmo Romão. Av. Bandeirantes, 3900. Campus Universitário, Monte Alegre, Ribeirão Preto (SP) CEP 14049-900 Fone: (0XX16) 602-2523.

\section{INTRODUÇ̃̃O}

A endoftalmite constitui uma das complicações mais graves e de pior resultado funcional entre as afecções oftalmológicas. Esta complicação é bastante rara após procedimentos cirúrgicos eletivos $(0,18 \%$ pós-cirurgia de catarata ${ }^{2}$ e $0,28 \%$ pós-transplante de córnea ${ }^{4}$ ), sendo um pouco mais freqüente em situações pós-traumatismo $(4,2 \%)^{5}$; o que seria explicado devido às condições de assepsia, desorganização das estruturas oculares e maior virulência dos agentes presentes no trauma.

$\mathrm{O}$ rápido e correto diagnóstico é fundamental para um tratamento adequado e precoce, mas pode ser confundido nos casos de trauma devido às alterações morfológicas e inflamatórias decorrentes deste ${ }^{1,5}$.

A escolha do tratamento é dificultada tendo-se em vista o tempo necessário para a identificação do agente etiológico e sua susceptibilidade às drogas. Com isto, a terapia é iniciada de maneira empírica, baseada em estudos realizados, em sua maioria, em outras localidades, não sendo, portanto, correspondentes à flora regional. Além disso, cumpre ressaltar a ocorrência de organismos resistentes às drogas utilizadas ${ }^{6} \mathrm{e}$ a dificuldade de obtenção de concentrações efetivas e duradouras destas drogas nas estruturas oculares. 
Este estudo objetivou caracterizar os casos de endoftalmite atendidos no HCFMRP-USP no intuito de melhor direcionar o tratamento e obter um resultado funcional mais satisfatório.

\section{MATERIAIS E MÉTODOS}

O estudo foi realizado através de uma análise retrospectiva dos casos de endoftalmite atendidos no HCFMRP-USP, no período de janeiro de 1993 a janeiro de 1998. O HCFMRP-USP constitui um hospital de nível terciário dentro do Sistema Único de Saúde do Ministério da Saúde vigente no país, sendo um centro de referência para o tratamento de casos oftalmológicos complexos, atendendo a toda região de Ribeirão Preto e outros estados, como Minas Gerais, Bahia e Mato Grosso do Sul.

Foram incluídos no estudo os pacientes que tinham o diagnóstico de endoftalmite, independente da causa do quadro, totalizando 58 pacientes. O prontuário de cada paciente foi analisado e os seguintes dados obtidos: a causa predisponente, sinais e sintomas, conduta, agente etiológico, tratamento e resultado funcional.

O resultado das culturas foi avaliado segundo os métodos laboratoriais padronizados internacionalmente ${ }^{12,17,21}$. Os meios utilizados foram: ágar-sangue, ágar-chocolate, tioglicolato e Sabouraud.

\section{RESULTADOS}

No período de janeiro de 1993 a janeiro de 1998 foram atendidos 58 casos de endoftalmite no HCFMRP-USP, sendo 45 pacientes do sexo masculino $(77,58 \%)$ e 13 do sexo feminino $(22,41 \%)$ (relação $3,46: 1)$.

O quadro de endoftalmite se desenvolveu após cirurgia em 23 casos $(39,65 \%)$, trauma em 23 casos $(39,65 \%)$, úlcera de córnea infectada em $7(12,06 \%)$ e um paciente $(1,72 \%)$ apresentou queimadura por carvão; em outros 4 pacientes não foi possível identificar a causa. Dos pacientes submetidos à cirurgia o procedimento realizado foi facectomia $(82,60 \%, 19 /$ $23)$, transplante de córnea $(8,69 \%, 2 / 23)$, glaucoma $(4,34 \%$, $1 / 23$ ) e retinopexia pneumática $4,34 \%, 1 / 23)$. Os traumas foram perfurantes em $60,86 \%(14 / 23)$ dos casos, contundentes em $30,43 \%(7 / 23)$ ou ambos em $8,69 \%(2 / 23)$.

O tempo para o início dos sintomas, a partir do fator desencadeante, foi de $1-3$ dias em $41,37 \%$ dos casos, $4-7$ dias em 13,79\%, 8-10 dias em 6,89\% e 10-30 dias em 30,48\%; em dois casos não foi possível definir este tempo.

Os sinais e sintomas mais freqüentemente observados foram, respectivamente, dor ocular $(74,13 \%)$, diminuição da visão $(63,79 \%)$, presença de secreção conjuntival $(32,75 \%)$ e fotofobia (8,62\%); e hiperemia conjuntival $(81,03 \%)$, hipópio $(56,89 \%)$, edema de córnea $(55,17 \%)$, secreção conjuntival purulenta (39,65\%), edema palpebral $(31,03 \%)$ e quemose $(18,96 \%)$.

O exame subsidiário predominantemente adotado foi a coleta de material para cultura $(70,48 \%)$, em $6,89 \%$ dos casos foi solici- tado ultra-som (US) de órbita e em 6 casos (10,34\%) tomografia computadorizada (TC) de órbita; em $24,13 \%$ dos casos nenhum exame foi solicitado. $\mathrm{O}$ resultado da cultura foi positivo em 35 casos $(85,36 \%)$ e negativo em $6(14,63 \%)$; em 5 casos houve coleta de vários locais sendo o resultado positivo em alguns e negativo em outros. Destaca-se o maior grau de positividade das culturas de vítreo $(83,3 \%)$ em relação às de líquido aquoso $(60 \%)$.

Os agentes etiológicos identificados estão mostrados na tabela 1. Geralmente apenas um agente etiológico foi isolado $(77,14 \%)$, dois agentes foram encontrados em $14,28 \%$, três em $5,70 \%$ e quatro em $2,85 \%$.

O antibiograma (Tabela 2), revelou dados importantes das características da flora regional: $100 \%$ dos Staphylococcus aureus foram resistentes à penicilina e ampicilina, mas $100 \%$ sensíveis às drogas mais usadas no tratamento (cefalotina e gentamicina); o Staphylococcus epidermidis mostrou certa resistência à gentamicina, mas foi $100 \%$ sensível à cefalotina; a Pseudomonas aeruginosa apresentou grande resistência à maioria das drogas utilizadas, porém $100 \%$ das cepas foram sensíveis à ceftazidima e $75 \%$ ao aztreonam; sendo que duas cepas mostraram-se sensíveis somente a estas drogas. Concordando com estudos da literatura, nenhum agente mostrou-se resistente à vancomicina ou ceftazidima.

A terapêutica foi tópica em $79,31 \%$ dos casos, endovenosa em $82,95 \%$, intra-vítrea em $6,89 \%$, oral em $6,89 \%$ e intramuscular em 3,44\%. O antibiótico predominantemente utilizado na terapia tópica foi cefalotina+gentamicina $(43,47 \%)$, cefalotina+amicacina $(21,73 \%)$, cefalotina isolado $(19,56 \%)$; na terapia endovenosa cefalotina+gentamicina $(47,91 \%)$, cefalotina+amicacina $(18,75 \%)$, cefalotina isolado $(16,67 \%)$; e na terapia intra-ocular vancomicina+amicacina $(50 \%)$, vancomicina $(25 \%)$ e cefalotina + gentamicina $(25 \%)$.

O tratamento cirúrgico consistiu em enucleação ou evisce-

Tabela 1 - Agentes etiológicos segundo causa predisponente

\begin{tabular}{|c|c|c|c|c|c|}
\hline \multirow{3}{*}{$\begin{array}{l}\text { Agente } \\
\text { (n.1) }\end{array}$} & & \multicolumn{4}{|c|}{ Causa(n.2) } \\
\hline & & \multirow{2}{*}{$\begin{array}{c}\text { Cirurgia } \\
\text { (18) }\end{array}$} & \multirow{2}{*}{$\begin{array}{c}\text { Trauma } \\
\text { (18) }\end{array}$} & \multirow{2}{*}{$\begin{array}{l}\text { Úlcera de } \\
\text { córnea } \\
\text { (7) }\end{array}$} & \multirow{2}{*}{$\begin{array}{c}\text { Outros } \\
\text { (3) }\end{array}$} \\
\hline & & & & & \\
\hline S.aureus & (12) & 7 & 3 & 2 & - \\
\hline S.epidermidis & (7) & 2 & 4 & 1 & - \\
\hline P.aeruginosa & (6) & - & 2 & 3 & 1 \\
\hline S.viridans & (5) & 3 & 2 & - & - \\
\hline E.coli & (3) & - & 2 & - & 1 \\
\hline Strepto $\beta$-hemolítico & $(2)$ & 1 & 1 & - & - \\
\hline E.cloacae & (2) & 1 & - & 1 & - \\
\hline S.pneumoniae & (2) & - & 1 & - & 1 \\
\hline P.mirabilis & (1) & 1 & - & - & - \\
\hline B.subtillis & (1) & - & 1 & - & - \\
\hline Corynebacterium sp & $(1)$ & - & 1 & - & - \\
\hline Streptococcus sp & (1) & - & 1 & - & - \\
\hline Fungos & (3) & 3 & - & - & - \\
\hline
\end{tabular}

N.1: número de cepas isoladas; N.2: número de casos em que foram isolados agentes na cultura; Outros: queimadura ou causa não identificada 


\begin{tabular}{|c|c|c|c|c|c|c|c|}
\hline \multirow{3}{*}{$\begin{array}{l}\text { Antibiótico } \\
\text { Sensibilidade \% }\end{array}$} & \multicolumn{7}{|c|}{ Agente etiológico ( $\mathrm{n}^{\circ}$ cepas) } \\
\hline & S.aureus & S.epidermidis & P.aeruginosa & S.viridans & E.coli & B.subtillis & E.cloacae \\
\hline & (12) & (6) & (6) & (5) & (3) & (1) & (2) \\
\hline Penicilina G & $0(12)$ & $40(5)$ & - & $100(5)$ & - & $0(1)$ & - \\
\hline Vancomicina & $100(12)$ & $100(6)$ & - & $100(5)$ & - & $100(1)$ & - \\
\hline Amicacina & $100(12)$ & $100(5)$ & $66,6(6)$ & $20(5)$ & $100(3)$ & $100(1)$ & $100(2)$ \\
\hline Ampicilina & $0(12)$ & $40(5)$ & $0(6)$ & $100(5)$ & $33,3(3)$ & $0(1)$ & $0(2)$ \\
\hline Cefalotina & $100(12)$ & $100(6)$ & $0(6)$ & $100(5)$ & $66,6(3)$ & $0(1)$ & $0(2)$ \\
\hline Gentamicina & $100(12)$ & $83,3(6)$ & $60(5)$ & $40(5)$ & $100(3)$ & $100(1)$ & $50(2)$ \\
\hline Tobramicina & $100(12)$ & $66,6(6)$ & $50(6)$ & $0(4)$ & $100(3)$ & $100(1)$ & $50(2)$ \\
\hline Imipenem & $100^{*}(4)$ & $100^{*}(2)$ & $66,6(3)$ & $50^{*}(2)$ & - & - & - \\
\hline Ceftazidima & $100^{*}(4)$ & $100(2)$ & $100(3)$ & $100^{*}(2)$ & - & - & - \\
\hline Ciprofloxacina & $100^{*}(5)$ & $75(4)$ & $60(5)$ & $100(3)$ & $100^{*}(1)$ & - & $100(1)$ \\
\hline Aztreonam & - & - & $75(4)$ & - & - & - & - \\
\hline Teicoplanina & $100(3)$ & $83,3(1)$ & - & - & - & - & - \\
\hline
\end{tabular}

ração, sendo o primeiro realizado em 13 pacientes $(24,41 \%)$ e indicada, mas ainda não realizada em 4 (6,89\%); a enucleação foi efetuada em 4 casos $(6,89 \%)$.

O resultado funcional (Gráfico 1) foi ausência de percepção luminosa em $65,51 \%$, percepção luminosa em $8,62 \%$, contagem de dedos em $3,44 \%$ e acuidade visual melhor que $0,3 \mathrm{em}$ $5,16 \%$; em 17,25\% dos casos não há referência a este dado.

\section{DISCUSSÃO}

Através deste estudo foi possível se obter dados importantes a respeito das características regionais dos casos de endoftalmite, como os sintomas e agentes etiológicos predominantes, o que certamente auxiliará no direcionamento adequado do tratamento e na obtenção de um melhor prognóstico.

Observamos uma predominância do sexo masculino $(77,58 \%)$, resultando numa proporção de 3,46: 1, que também é observado em outros estudos ${ }^{2,5,13}$; sendo que nestes a proporção é maior nos casos pós-traumáticos, justificado pelo maior risco das profissões exercidas pelo sexo masculino ${ }^{13}$.

Os dois principais fatores desencadeantes de endoftalmite (trauma e cirurgia) apresentaram um número igual de casos: $23(39,45 \%)$, diferindo da literatura que revela uma predominância de casos pós-cirúrgicos $\left(60 \%{ }^{8} ; 66,2 \%{ }^{24}\right)$ em relação aos pós-traumáticos $\left(25 \%^{1} ; 20 \%{ }^{8} ; 25,4 \%{ }^{24}\right)$.

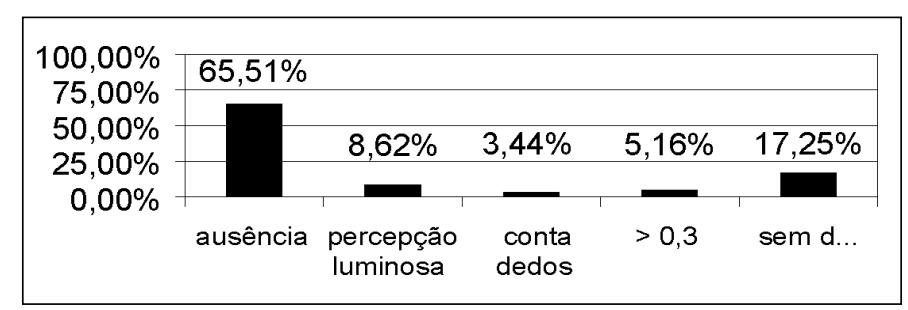

Gráfico 1 - Resultado Funcional
O cálculo do risco específico de endoftalmite não foi possível de ser efetuado, visto que não se possuía informações precisas a respeito do local onde o paciente havia sido submetido ao procedimento cirúrgico e também se havia buscado atendimento oftalmológico em outro serviço e, conseqüentemente, sofrido alguma manipulação prévia das estruturas oculares.

Apesar dos padrões e métodos utilizados para se obter a melhor assepsia nos procedimentos cirúrgicos vários estudos comprovaram a contaminação de estruturas oculares durante o procedimento ${ }^{3,16,22}$. A principal fonte de infecção é a flora do paciente (pálpebra, nariz, conjuntiva), sendo os organismos destas localidades geneticamente semelhantes aos encontrados nas culturas de vítreo ${ }^{16}$. Além disso, foi observado um aumento no risco de contaminação de acordo com o fato do cirurgião falar durante o procedimento e do número de pessoas na sala ${ }^{31}$. O uso de iodeto de povidine tópico e antibioticoterapia no pré-cirúrgico também é apregoado na redução da contaminação ${ }^{32-35}$. Nos casos de implante de lente intraocular, deve-se dar preferência ao polimetilmetacrilato, por ser um material que apresenta menor risco de colonização por organismos patogênicos ${ }^{32}$.

A presença de corpo estranho nos casos pós-traumáticos é responsável por uma incidência maior de infecção $\left(15 \%{ }^{5}\right.$, $10 \%^{1}, 6,9 \%{ }^{13}$ ), apesar da composição do material não alterar o prognóstico.

Em transplantes de córnea a realização de culturas do material do doado não é recomendada, tendo-se em vista o custo e o baixo valor preditivo obtido desta padronização ${ }^{4}$.

A análise do tempo para o início dos sintomas revelou que em $41,37 \%$ dos casos este ocorreu nas primeiras 72 horas e em $34,48 \%$ entre 10-30 dias após o fator desencadeante. Os demais casos surgiram entre 4-9 dias. Outros estudos mostram uma incidência maior no período 2-7 dias pós evento ${ }^{7,15,25,30}$. Em nosso meio vários fatores levam à demora na obtenção de atendimento e tratamento: aspectos culturais (religião, medo, 
desinformação, desinteresse) e sócio-econômicos (ausência ou lotação de serviço disponível, ausência de meio de locomoção). A baixa virulência do agente etiológico, predominante nos casos pós-catarata, também contribui para uma busca tardia por atendimento ${ }^{32}$. O tempo para qual é marcado o retorno no póscirúrgico tem se mostrado muito extenso com os sintomas surgindo até 1,9 dias antes da data marcada ${ }^{25}$, comprometendo acentuadamente o prognóstico, que é melhor quando se efetua um atendimento precoce ${ }^{13,19,25,35}$.

Os sinais e sintomas predominantes foram semelhantes aos descritos, havendo apenas pequenas variações ${ }^{12,15,30,32,34}$. A presença de dor, principal queixa dos pacientes $(74,13 \%)$, foi semelhante à taxa referida em outro estudo $(74 \%)^{12}$ e sua presença ou ausência não está relacionada ao resultado microbiológico ${ }^{12,21}$. Os sinais presentes foram hiperemia conjuntival $(81,03 \%)$, hipópio $(56,89 \%)$, edema de córnea $(57,17 \%)$, secreção conjuntival $(39,45 \%)$ e edema palpebral $(31,03 \%)$. A presença de edema palpebral foi relacionada com maior taxa de infecção por organismos Gram positivos ${ }^{10}, \mathrm{o}$ que não foi observado neste estudo. A avaliação do vítreo e fundo de olho não foi realizada na maioria dos casos, não sendo, portanto, consideradas no estudo.

Dentre os exames subsidiários disponíveis utilizou-se a coleta de material para a cultura em $70,48 \%$ dos casos, tomografia computadorizada em $10,34 \%$ e ultra-som em $6,89 \%$; em $24,13 \%$ não foi solicitado nenhum exame subsidiário. Os exames de TC e US foram realizados nos casos de trauma com suspeita de corpo estranho intra-ocular, considerando-se o maior risco de infecção na sua presença ${ }^{1,5,13}$. Estes exames mostraram, além do corpo estranho quando presente, alterações estruturais devido à infecção como aumento de volume de partes moles e intenso reforço pós-contraste.

O material colhido para cultura foi secreção conjuntival $(53,44 \%)$, aquoso $(17,24 \%)$, vítreo $(10,30 \%)$ e córnea $(8,62 \%)$. O resultado foi positivo em $85,36 \%$ das culturas, um índice um pouco acima dos dados da literatura $\left(62 \%{ }^{8}, 65 \%{ }^{13}\right.$, $73 \%{ }^{15}, 39 \%{ }^{24}, 60 \%{ }^{26}$ ). O vítreo mostrou um índice de positividade maior que o aquoso $(83,30 \%$ a $60,00 \%)$, confirmando dados da literatura ${ }^{1,7,19}$, que também revelam o fato de, em alguns casos, ele ser a única fonte de cultura positiva ${ }^{18}$. Tal fato pode ser resultado de uma melhor limpeza do aquoso devido ao seu maior fluxo e celularidade ${ }^{33,38}$. Além disto, outro dado preocupante diz respeito à positividade de culturas de meios tidos como estéreis pelo oftalmologista (até $33 \%$ ) $^{8} \mathrm{e}$ ao fato da conduta terapêutica poder ser alterada após o resultado da cultura ${ }^{27}$, visto que neste estudo em $24,13 \%$ dos casos não houve realização de nenhum exame subsidiário. Outro erro foi o grande percentual de casos em que a secreção conjuntival foi o único material coletado, ao contrário do recomendado: obtenção de amostras do esfregaço conjuntival, punção do humor aquoso e corpo vítreo ${ }^{34}$.

O agente etiológico mais freqüente foi o Staphylococcus aureus, seguido do Staphylococcus epidermidis e Pseudomonas aeruginosa, diferindo de outros estudos que revelam maior freqüência do S. epidermidis ${ }^{7,8,15,19,33}$; apenas outro estudo ${ }^{38}$ revelou maior freqüência deste agente. Contudo, ao se analisar a relação com a causa predisponente observamos que nos casos pós-traumáticos o S.epidermidis é o agente mais freqüente, semelhante a outros estudos ${ }^{1,5}$, apesar de haver somente um caso $(5,55 \%)$ de Bacillus, enquanto que nestes estudos este é o segundo mais freqüente apresentando um péssimo prognóstico visual ${ }^{7,32}$.

Nos casos pós-cirúrgicos o agente mais observado foi o S.aureus, ao contrário da literatura ${ }^{3,15,16,26}$ que revela predomínio do S.epidermidis, sendo que neste estudo houve apenas um caso $(5,55 \%)$ por este agente. O $S$. aureus também é o mais observado em outro estudo ${ }^{24}$. Tal fato poderia ser explicado pela baixa virulência do S. epidermidis e uma maior participação do $S$. aureus na flora dos indivíduos estudados. Outro dado interessante é a alta incidência de fungos (16,66\%), sendo dois casos de Scedosporium apiospermum e um de Fusarium $s p$, visto que esta incidência era esporádica em outros estudos 18, 19 , além do fato dos agentes observados serem Candida sp e Aspergillus $s p{ }^{28}$. Este dado mostra-se importante por identificar uma possível falha na assepsia, como o uso de soluções contaminadas ${ }^{34}$, e reforçar a necessidade de meio de cultura adequado para sua identificação ${ }^{18} \mathrm{e}$ tratamento direcionado. Nos três casos a acuidade visual final foi ausência de percepção luminosa.

$\mathrm{O}$ agente mais freqüente nos casos de complicações em úlceras de córnea foi a $P$. aeruginosa $(42,80 \%)$, seguida do $S$. aureus $(28,57 \%)$. Em estudos realizados em casos de endoftalmite por agentes Gram negativos a $P$. aeruginosa foi o principal agente $(23 \%)$, sendo a responsável pelos casos pósúlcera de córnea ${ }^{12,22}$. Tal fato é de extrema importância devido ao surgimento de resistência a antibióticos nesta espécie $^{14,20}$ (Tabela 2) e do mau prognóstico visual ${ }^{8,17}$.

Os Streptococcus sp foram responsáveis por aproximadamente $20 \%$ dos casos e estão relacionados com um prognóstico pior que os S. epidermidis ${ }^{8,23,26}$. Em nosso estudo, todos os cinco casos de $S$. viridans evoluíram para ausência de percepção luminosa.

O S. epidermidis mostrou-se o menos virulento dos agentes, com 57,14\% dos casos apresentando acuidade visual melhor que percepção luminosa.

O tratamento baseou-se principalmente em terapia tópica $(79,31 \%)$ e endovenosa $(82,75 \%)$, normalmente associados e com uso de cefalotina e gentamicina ou amicacina na maioria dos casos. O uso intra-ocular foi efetuado em apenas $6,89 \%$ dos casos, sendo a vancomicina a droga mais utilizada. Nos casos de agentes fúngicos a droga utilizada foi a anfotericina $\mathrm{B}$ por via endovenosa. Vitrectomia total ou parcial não foi realizada em nenhum caso. Tais condutas não obedecem aos estudos recentes no que tange à melhor acuidade visual final com o uso da vitrectomia ${ }^{21,34}$; a concentração vítrea abaixo da mínima inibitória de cefalotina via endovenosa (exceto para S. epidermidis) ${ }^{10,11}$; ao surgimento de resistência a esta droga ${ }^{20,35}$; à toxicidade macular do aminoglicosídeo quando usado intravítreo ${ }^{6,34,35}$, à concentração vítrea atingida (abaixo da mínima inibitória) quando via endovenosa e a resistência surgida às drogas desta classe ${ }^{6,17,32,35}$. A efetividade da ciprofloxacina via 
oral foi observada contra $S$. epidermidis, Bacillus $s p$ e Enterobacter $s p$, mas não para $S$. aureus e $P$. aeruginosa ${ }^{11}$. Desta forma, os estudos revelam que o tratamento mais eficaz considerando-se a concentração atingida pela droga nas estruturas oculares, o espectro alcançado e a baixa toxicidade é o uso de vancomicina e ceftazidima via intra-ocular $6,7,9,17,21,23,29,35,36$ e o uso de vitrectomia em casos selecionados ${ }^{9,21,34}$. O uso de esteróides, ainda controverso, não foi abordado neste estudo.

O resultado funcional (Gráfico 1) foi bastante desfavorável, confirmando a perda visual severa nos casos de endoftalmite. Contudo, estes resultados foram bastante piores que os relatados $5,7,13,17,19,21$ levando-nos a analisar os fatores responsáveis pelos mesmos e as condutas a serem adotadas para uma mudança no quadro.

Algumas sugestões seriam a padronização da coleta para cultura de líquido conjuntival, aquoso e vítreo em todos os casos suspeitos de endoftalmite; uso de meio adequado para cultura de fungos; melhora da assepsia nos procedimentos cirúrgicos; diminuição do tempo de retorno após cirurgia; promoção do tratamento com vancomicina + ceftazidima via intra-ocular e realização de vitrectomia em casos selecionados; prevenção de acidentes e tratamento das condições que comprometam o estado imunológico do paciente, como desnutrição, diabetes alcoolismo e doenças imunitárias.

Agradecimentos: Ao Serviço de Arquivo Médico do HCFMRP-USP pelo acesso aos prontuários dos pacientes.

\section{SUMMARY}

Purpose: 58 cases with a diagnosis of endophthalmitis were reviewed in the attempt to obtain regional characteristics of this pathology. Predisposing factors, clinical presentation, ancillary examination, treatment and functional results were analyzed.

Methods: Analysis of charts of patients treated in the Clinical Hospital of Ribeirão Preto's Medical School (USP), from 1993 to 1998.

Results: Trauma and surgery were the most frequent causes (39.65\% each one). The most common clinical presentation was pain and decrease of visual function associated with hypopyon and conjunctival injection. In $70.48 \%$ of cases samples for culture were collected and $85.36 \%$ of the specimens were positive. The most frequent microorganism was S. aureus (26.08\%), followed by S. epidermidis and $\mathrm{P}$. aeruginosa (13.04\% each one). All isolated species were susceptible to vancomicyn and ceftazidime. In $65.51 \%$ of the cases no light perception was the final visual acuity.

Conclusions: These results suggest some changes that might be performed to improve prognosis of endophthalmitis, such as using intraocular vancomicyn and ceftazidime.

\section{REFERÊNCIAS BIBLIOGRÁFICAS}

1. Moreira CA. Endoftalmite traumática. Biblioteca Brasileira de Oftalmologia. Ed. Cultura Médica, Rio de Janeiro, 1994;17:136-42.

2. Norregaard JC, Thoning H, Bernth-Petersen P et al. Risk of endophthalmitis after cataract extraction: results from the International Cataract Surgery Outcome study. Br J Ophthalmol 1997;81:102-6.

3. Mistlberger A, Ruckhofer J, Raithel E et al. Anterior chamber contamination during cataract surgery with intraocular lens implantation. J Cataract Refract Surg 1997;23:1064-9.

4. Wiffen SJ, Weston BC, Maguire LJ, Bourne WM. The value of routine donor corneal rim culture in penetrating keratoplasty. Arch Ophthalmol 1997; 115:719-24.

5. Duch-Samper AM, Menezo JL, Hurtado-Sarrió. Endophthalmitis following penetrating eyes injuries. Acta Ophthalmol Scand 1997;75:104-6.

6. Roth DB, Flynn HW. Antibiotic selection in treatment of endophthalmitis: the significance of drug combination and synergy. Surv Ophthalmol 1997;41:395-401.

7. Mao LK, Flynn HW, Miller D, Pflugfelder SC. Endophthalmitis caused by Staphylococcus aureus. Am J Ophthalmol 1993;116:584-9.

8. Bohigian GM, Olk RJ. Factors associated with poor visual result in endophthalmitis. Am J Ophthalmol 1986;101:332-4.

9. Aguilar HE, Meredith TA, Drew C et al. Comparative treatment of experimental Staphylococcus aureus endophthalmitis. Am J Ophthalmol 1996;121:310-7.

10. Axelrod JL, Klein RM, Bergen RL, Sheikh MZ. Human vitreous level of selected antistaphylococcal antibiotics. Am J Ophthalmol 1985;100:570-5.

11. El baba FZ, Trousdale MD, Gauderman WJ, Wagner DG, Liggett PE. Intravitreal penetration of oral ciprofloxacin in humans. Ophthalmology 1992; 99:483-6.

12. Johnson MW, Doft BH, Kelsey SF et al. The endophthalmitis vitrectomy study: Relationship between clinical presentation and microbiologic spectrum. Ophthalmology 1997;104:261-72.

13. Thompson JT, Parver LM, Enger CL et al. Infectious endophthalmitis after penetrating injuries with retained intraocular foreign bodies. Ophthalmology 1993;100:1468-74.

14. Murray BE. Can antibiotic resistence be controlled? N Engl J Med 1994;330:1229-30.

15. Fisch A, Salvanet A, Prazuck T et al. Epidemiology of infective endophthalmitis in France. Lancet 1991;38:1373-6.

16. Speaker MG, Milch FA, Shah MK, Eisner W, Kreiswirth BN. Role of external bacterial flora in the pathogenesis of acute postoperative endophthalmitis. Ophthalmology 1991;98:639-49.

17. Irvine WD, Flynn HW Jr, Miller D, Pflugfeder SC. Endophthalmitis caused by gram-negatives organisms. Arch Ophthalm 1992;110:1450-4.

18. Barza M, Pavan PR, Doft BH et al. Evaluation of microbiological techniques in postoperative endophthalmitis in the endophthalmitis vitrectomy study. Arch Ophthalm 1997;115:1142-50.

19. Puliafito CA, Baker AS, Haaf J, Foster CS. Infectious endophthalmitis: review of 36 cases. Ophthalmology 1982;89:921-9.

20. Flynn HW, Pulido JS, Plugfeder SC et al. Endophthalmitis therapy: changing antibiotic sensitivity patterns and current therapeutic recommendations. Arch ophthalm 1991;109:175-6.

21. Endophthalmitis vitrectomy study group. Results of the Endophthalmitis vitrectomy study: a randomized trial of immediate vitrectomy and intravenous antibiotics for the treatment of postoperative bacterial endophthalmitis. Arch Ophthalm 1995;113:1479-96.

22. Whitcher JP. Editorials. The treatment of endophthalmitis - still an exercise in frustation. Br J Ophthalmol 1997;81:713-5.

23. Mao LK, Flynn HW, Miller D, Pflugfeder SC. Endophthalmitis caused by Streptococcal species. Arch Ophthalm 1992;110:798-801.

24. Chaib AR, Freitas D, Scarpi MJ, Guidugli T. Pesquisa laboratorial em endoftalmite. Arq Bras Oftalmol 1997;60:250-7.

25. Lam SR, Tuli R, Menezes A, Devenyi RG. NB. Bacterial endophthalmitis following extracapsular cataract extraction: recommendations for early detection. Can J Ophthalm 1997;32:311-4.

26. Somani S, Grinbaum A, Slomovic AR. Postoperative endophthalmitis: incidence, predisposing surgery, clinical course and outcome. Can J Ophthalm 1997;32:303-10.

27. Rubsamen PE, Cousins SW, Martinez JÁ. Impact of cultures on managment decisions following surgical repair of penetrating ocular trauma. Ophthalmic Surg Lasers 1997;28:43-9.

28. Essman TF, Flynn HW Jr, Smiddy WE et al. Treatment outcomes in a ten-year 
study of endogenous fungal endophthalmitis. Ophthalmic Surg Lasers 1997;28:185-94.

29. Schech JM, Alfaro DV, laughlin RM, Sanford EG, Briggs J Dalgetty M. Intravenous gentamicin and ceftazidime in penetrating ocular trauma: a swine model. Retina 1997;17:28-32.

30. Alves AA Jr, Motta MMS. Endoftalmite bacteriana pós-facectomia. Diagnóstico, tratamento e profilaxia. Rev Bras de Oftalmol 1987;46:32-6.

31. Lagos C, Bazzi GF, Dziecinny LH, Moreira H, Repka JC, Cabrera PA. Análise do risco de contaminação em cirurgias oftalmológicas. Rev Bras de Oftalmol 1995;54:55-8.

32. Freda R, Gama ADJC. Endoftalmite: revisão. Rev Bras de Oftalmol 1995;54:35-40.
33. Alves AA Jr, Cardozo AV, Valle RR. Incidência de endoftalmite infecciosa pós-facectomia no HSE. Análise de fatores de risco e medidas profiláticas. Rev Bras de Oftalmol 1995;54:29-33.

34. Molinari LC, Peyman GA. Guia prático para o manejo da endoftalmite. Rev Bras de Oftalmol 1996;55:7-18.

35. Molinari LC. Endoftalmites: aspectos profiláticos e terapêuticos. Rev Bras de Oftalmol 1995;54:37-43.

36. Coelho RS, Monaretto A, Germanos CRA. Endoftalmite crônica do pseudofácico: relato de caso atípico e discussão. Rev Bras de Oftalmol 1995;54:61-4.

37. Kwitko S, Melamed J, Barcaro S, Verri JM, Petrillo VF. Endoftalmite endógena por Listeria monocytogenes. Arq Bras Oftalmol 1993;56:326-9.

\section{CONGRESSO LATINO-AMERICANO DE ESTRABISMO II a I4 de Maio de 2000 Maksoud Plaza Hotel - São Paulo}

\section{Promoção}

Conselho Latino-americano de Estrabismo

\section{Apoio}

Conselho Brasileiro de Oftalmologia

\section{Comissão Executiva}

Carlos Fumiaki Uesugui

Carlos R. de Souza-Dias

Luís Eduardo M. R. de Carvalho
Mauro Goldchmit

Rita de Cássia V. Damiani

Rosana N. Pires da Cunha

\section{Convidados Internacionais}

\section{Alfonso Castanera de Molina - Barcelona - Espanha}

- Diretor do Instituto de Oftalmologia Pediátrica de Barcelona

\section{Creig S. Hoyt - São Francisco - EUA}

- Professor de Oftalmologia Pediátrica da Universidade da Califórnia
Leslie France - Madison - EUA

- Ortoptista do Setor de Oftalmologia Pediátrica \& Estrabismo - Universidade de Wisconsin

Nélida B. Melek - Buenos Aires - Argentina

- Professora Adjunta da Universidad del Salvador

\section{Informações: CBO Eventos}

Alameda Santos, 1343 - Cj. I. II 0

CEP 0I4I9-00I - S. Paulo - SP

Telf.(xx I I) - 3266-4000 - Fax: (xx I I) 3I7I-0948 